$$
\text { Mary Ann Lielest, Inc. publishers }
$$

\title{
Willingness to Participate in Longitudinal Research Among People with Chronic Pain Who Take Medical Cannabis: A Cross-Sectional Survey
}

\author{
Marcus A. Bachhuber, Julia H. Arnsten, Joanna L. Starrels, and Chinazo O. Cunningham
}

\begin{abstract}
Background: Regulatory barriers limit clinical trials of medical cannabis in the United States. Longitudinal cohort studies may be one feasible alternative that could yield clinically relevant information. Willingness to participate in such studies is not known.

Materials and Methods: In October 2016, we surveyed a convenience sample of patients with chronic pain from two New York registered organizations (responsible for growing, processing, distributing, and retailing medical cannabis products). After a vignette describing a longitudinal cohort study involving weekly patientreported outcomes and quarterly assessments of physical functioning and urine and blood tests, we asked about respondents' willingness to participate. We examined willingness to participate, duration of participation, and frequency of data collections overall and by subgroups, using multivariable logistic regression models.

Results: Of 405 respondents (estimated response rate: 30\%), 54\% were women and 81\% were white nonHispanic. Neuropathy was the most common pain condition (67\%) followed by inflammatory bowel disease (19\%). Of respondents, 94\% (95\% Cl 92-97\%) thought that the study should be done, 85\% (95\% Cl 81-88\%) would definitely or probably enroll if asked, $76 \%$ (95\% Cl 72-81\%) would participate for $\geq 1$ year, and 59\% (95\% Cl 54-64\%) would respond to questions at least daily. Older age was the only factor associated with lower willingness to participate, lower willingness to participate for $\geq 1$ year, and lower willingness to respond to questions at least daily.

Conclusions: Nearly all respondents were supportive of the proposed study and most reported that they would enroll if asked. Enhanced engagement with older individuals may be needed to promote equal enrollment. Recruitment for longitudinal cohort studies with frequent data collection appears feasible in this patient population.
\end{abstract}

Keywords: medical marijuana; marijuana; chronic pain; cohort studies; analgesics

\section{Introduction}

Chronic pain is common in the United States, and its management is challenging. Over the past two decades, opioid analgesics have become a leading pain management strategy and dispensing has tripled. ${ }^{1}$ In parallel, the incidence of opioid use disorder and opioid overdoses have both dramatically increased. ${ }^{1,2}$ To reduce these harms, patient groups,

Division of General Internal Medicine, Department of Medicine, Albert Einstein College of Medicine/Montefiore Medical Center, Bronx, New York.

*Address correspondence to: Marcus A. Bachhuber, MD, MSHP, Division of General Internal Medicine, Department of Medicine, Albert Einstein College of Medicine/ Montefiore Medical Center, 3300 Kossuth Avenue, Bronx, NY 10467, E-mail: marcus.bachhuber@gmail.com

(C) Marcus A. Bachhuber et al. 2018; Published by Mary Ann Liebert, Inc. This Open Access article is distributed under the terms of the Creative Commons License (http://creativecommons.org/licenses/by/4.0), which permits unrestricted use, distribution, and reproduction in any medium, provided the original work is properly cited. 
clinicians, and policymakers have called for new strategies to address pain management and reduce use of opioid analgesics. One important and rapidly expanding strategy to manage chronic pain is the use of medical cannabis. As of January 2018, medical use of cannabis is legal in 29 states and the District of Columbia.

In January 2017, the National Academies of Science, Engineering, and Medicine released a landmark report finding substantial evidence for cannabis' efficacy in treating chronic pain. ${ }^{3}$ But beyond evidence of efficacy, there are numerous gaps in research, including patient selection, long-term treatment outcomes, and dosing of major cannabinoids such as $\Delta 9$-tetrahydrocannabinol (THC) and cannabidiol (CBD). While randomized controlled trials (RCTs) are the "gold standard" of evidence, RCTs of medical cannabis (a Schedule I substance) currently face numerous hurdles in the United States both due to product availability and due to restrictive dispensing and security procedures required. Although the Drug Enforcement Administration announced a policy change to expand the number of cannabis manufacturers, currently only one entity is authorized to produce and supply cannabis to U.S. researchers. ${ }^{3,4}$ The cannabis products available for research are limited in scope and not necessarily comparable to cannabis products available in state dispensaries. ${ }^{3}$ Even when products are obtained for research, they typically must be dispensed in a directly observed setting. Because of these restrictions, over the last 41 years, only six clinical trials in the United States have administered cannabis to examine its effect on pain. These randomized trials all occurred in tightly controlled settings (human laboratories), for short durations ( $4 \mathrm{~h}$ to 12 days), with small sample sizes (10-55 participants). ${ }^{5-10}$

Randomized trials might also face several other challenges. Unlike a novel pharmaceutical, many people with chronic pain have used cannabis and may bring those experiences to a study, potentially influencing results. However, in contrast, enrolling participants who are cannabis-naive may not necessarily be representative of the broader patient population because of such widespread use. In addition, strong political views and a desire to advocate for medical cannabis access may lead to enrollment of participants who are invested in a certain outcome. Finally, people with chronic pain may not be motivated to enroll in a clinical trial because of cannabis' widespread availability outside of the trial.
Given current limitations of interventional research, observational studies are an appealing alternative. Longitudinal cohort studies of patient-reported pain outcomes are feasible, and even intensive assessments of pain (i.e., several times daily) have not been found to affect participants' responses. ${ }^{11,12}$ While longitudinal cohort studies that simply compare those who use medical cannabis to those who do not would be inescapably confounded, more complex designs and analyses could potentially come closer to estimating causation. ${ }^{13}$ From a clinical perspective, studying medical cannabis in a naturalistic setting may also be more aligned with how the system currently operates. Physicians do not prescribe medical cannabis such as other medications, but they certify patients to purchase it. Patients then choose the dose, amount, and route of administration.

Despite the urgent need for research on medical cannabis and chronic pain, willingness of the target population to participate in such research is not known. Frustration with previous treatment failures and a desire for new options may motivate patients to participate in this line of research. In contrast, experiences of stigma and marginalization from the healthcare system may dissuade patients from participating. ${ }^{14,15}$ The purpose of the current study is to examine willingness to participate in research among patients with chronic pain who take medical cannabis. Overall and by demographic (i.e., age and sex) and clinically important subgroups (e.g., taking opioid analgesics), we describe willingness to participate in a longitudinal study, duration for which potential participants would join in the study, and frequency with which potential participants would provide data.

\section{Materials and Methods}

Setting and population

The setting for the current study is New York State. New York State's medical cannabis program (operational in January 2016) has several features that make it a promising venue for longitudinal research on medical cannabis and chronic pain. To be certified for medical cannabis, individuals must have at least one qualifying condition (cancer, neuropathy, HIV/ AIDS, inflammatory bowel disease, spinal cord injury, multiple sclerosis, epilepsy, and several other neurological diseases) and one associated complication (severe or chronic pain, cachexia or wasting syndrome, severe nausea, seizures, or severe or persistent muscle spasms). After a program expansion in March 2017, after this 
study was conducted, patients with severe or chronic pain from any condition qualify (not just the conditions listed above). Patients can only purchase medical cannabis products from state-licensed dispensaries, which offer products with a variety of THC:CBD ratios with several different routes of administration (sublingual tincture, PO oil or capsule, and oil for vaporization)-whole plant material is not available in New York dispensaries. As all products are third-party tested for content and allow for dosing in milligrams, cannabis use can be measured with relative precision.

We surveyed customers of two New York State registered organizations (responsible for growing, processing, distributing, and retailing medical cannabis products). In October 2016, we recruited a convenience sample of patients with chronic pain by posting fliers in dispensaries, approaching patients in waiting rooms, and sending out an electronic link via patient newsletters. Recruitment materials specified that the survey was for patients with chronic pain, but did not specify that the topic would be willingness to participate in clinical research. Respondents were offered a \$15 gift card for completion of the survey. Inclusion criteria were as follows: (1) age $\geq 18$ years, (2) registration as a patient with the New York State medical cannabis program, (3) chronic or severe pain as a qualifying complication (by self-report), and (4) able to comprehend English. We excluded respondents who completed the survey in less than $90 \mathrm{sec}$. While we could not determine the exact number of eligible patients, we estimated that the two registered organizations served $30 \%$ of an estimated 6000 medical cannabis patients in New York at the time of the survey, 75\% of which have chronic or severe pain, ${ }^{16}$ for a total estimate of 1350 eligible patients.

\section{Survey}

We created a 28 -question survey to examine willingness to participate in longitudinal cohort studies among patients with chronic pain who take medical cannabis. Based on potential research participants' information needs as described in previous work, we created a vignette describing a hypothetical longitudinal cohort study involving patient-reported outcomes as well as assessments of physical functioning and urine and blood tests (Supplementary Data). ${ }^{17,18}$

After reading the description, we first asked respondents, "Do you think this study should be done?" (answer choices: definitely yes, probably yes, probably no, and definitely no). We then asked respondents' willing- ness to participate through a series of three questions: (1) "Would you participate in the study if you were asked?" (answer choices: definitely yes, probably yes, probably no, and definitely no), (2) "If you joined the study, would you be willing to participate for": (answer choices: 5 years, 2 years, 1 year, 6 months, 3 months, and I would not join the study), and (3) "If you were texted or prompted on your cell phone to answer questions from the study, how often would you be willing to respond?" (answer choices: twice or more daily, once daily, three times a week, once a week, twice a month, once a month, less than once a month, and I would not join the study). We drew the wordings and based our answer choices for these questions from a recent study. ${ }^{19}$

For respondents' indicating a willingness to participate in the proposed study (for any length of time), we presented a list of potential reasons why and asked participants to check all that apply. Similarly, for respondents not willing to participate, we displayed a list of potential reasons why not and asked respondents' to check all that apply. We drew these potential reasons from studies examining willingness to participate in diverse types of research. ${ }^{19-38}$

In addition to collecting information on respondents' willingness to participate, we collected information about respondents' sociodemographic characteristics, pain condition and medication use, and information about medical cannabis qualifying conditions and product use. For sociodemographic characteristics, we collected age, sex, race/ethnicity (Asian/pacific islander, black non-Hispanic, white non-Hispanic, Hispanic/ Latino of any race, and any other race or multiple races), education level, income, employment, and health insurance. For pain condition, we collected information on the duration of the pain condition and the frequency of pain, as well as a three-item measure of pain intensity and interference with functioning (the PEG scale). ${ }^{39} \mathrm{We}$ classified pain and interference scores from the PEG (mean of the three items) into mild, moderate, and severe using established cutoffs. ${ }^{40}$ For pain medication use, we asked about use of common medication types in the past 30 days, and for respondents reporting opioid analgesic use, we asked about the number of days used in the past 30 days. For medical cannabis information, we collected the number of months the respondent has used medical cannabis, number of days in the past 30 days used, dosage forms (high THC, balanced THC:CBD, high CBD), and routes of administration (sublingual, oral, vapor). 


\section{Missing data}

Of all respondents, $83 \%(n=338 / 405)$ provided complete data. To account for missing data, we conducted a multiple imputation procedure with chained equations. ${ }^{41}$ We performed this in stages. First, we imputed sociodemographic variables, followed by pain condition and medication use variables, then use of New York State medical cannabis products, and finally, willingness to participate in research. We assumed data were missing at random and created 20 imputed datasets. All data presented include $95 \%$ confidence intervals $(95 \% \mathrm{CI})$ to account for the uncertainty around the imputed data.

\section{Statistical analysis}

First, we calculated descriptive statistics (mean, median, frequencies) along with 95\% CIs for all survey questions. Next, to describe willingness to participate in research among specific subgroups, we used multivariable logistic regression models. Our main outcomes were the three questions on willingness to participate, and we dichotomized responses for these questions. For the question, "Would you participate in the study if you were asked?," we dichotomized responses as "definitely yes" and "probably yes" compared to "probably no" and "definitely no." For the question, "If you joined the study, would you be willing to participate for?" we dichotomized responses as " $\geq 1$ year" and " $<1$ year or would not participate." For the question, "If you were texted or prompted on your cell phone to answer questions from the study how often would you be willing to respond?" we dichotomized responses as "once or more daily" and "less than daily." We selected independent variables for our models based on factors previously found to be associated with willingness to participate (i.e., age, sex, race/ ethnicity, education) and variables that delineate clinically important subgroups (i.e., cancer versus noncancer pain, severe versus mild/moderate pain, interference with function, and use of opioid analgesics in the previous 30 days). ${ }^{18-21,23,24,29-37,42-46}$ To avoid small cell sizes, we collapsed levels of these categorical variables into clinically meaningful dichotomies. For each model, we estimated the predicted probability of the outcome (e.g., the percentage of women who would participate in the study if asked) using predictive margins. We also estimated contrasts (e.g., the difference in the percentage of women who would participate compared with men). Analyses were conducted using SAS 9.4 and Stata 13. This research was approved by the Albert Einstein College of Medicine and Montefiore Medical Center Institutional Review Board (protocol 2016-6728).

\section{Results}

Of 599 respondents, 191 (32\%) were excluded due to ineligibility (150 were not registered as patients with New York State, 37 did not report chronic pain as a symptom, and 4 did not consent), and $3(0.5 \%)$ were excluded for taking $<90 \mathrm{sec}$ to complete the survey. Based on our estimates of the number of eligible patients, this represents an estimated response rate of $30 \%(n=405 / 1350)$.

Sociodemographic characteristics of the final sample are shown in Table 1 . The majority were women (54\%, 95\% CI 49-59\%), white non-Hispanic (81\%, 95\% CI $77-85 \%)$, and had college or graduate degrees (35\%, $95 \%$ CI $30-40 \%$ and $25 \%$, 95\% CI 20-29\%, respectively).

\section{Table 1. Demographic and Clinical Characteristics of New York State Medical Cannabis Patients with Severe or Chronic Pain $(N=405)$}

\begin{tabular}{lc}
\hline Characteristic & $\%(95 \% \mathrm{CI})$ \\
\hline Age, median $(n=404)$ & $53(51-55)$ \\
Female gender $(n=405)$ & $54(49-59)$ \\
Race/ethnicity $(n=404)$ & \\
Asian/Pacific Islander & $1(0-2)$ \\
Black & $5(3-7)$ \\
Hispanic/Latino, of any race & $9(6-12)$ \\
Native American/Alaskan Native & $1(0.02-2)$ \\
White & $81(77-85)$ \\
Other/multiple races & $4(2-6)$ \\
Education $(n=352)$ & \\
Less than high school & $1(0.02-2)$ \\
High school diploma or GED & $12(8-15)$ \\
Some college & $27(23-32)$ \\
College degree & $35(30-40)$ \\
Graduate degree & $25(20-29)$ \\
Income $(n=342)$ & \\
Less than $\$ 20,000$ & $20(16-24)$ \\
\$20,000-\$39,000 & $15(12-19)$ \\
\$40,000-\$59,000 & $15(11-19)$ \\
\$60,000-\$79,000 & $11(7-14)$ \\
\$80,000 or higher & $39(34-44)$ \\
Work status $(n=354)$ & \\
Full time & $24(20-28)$ \\
Part time & $7(4-9)$ \\
Retired & $17(12-20)$ \\
Unemployed & $7(4-10)$ \\
Disabled & $46(41-51)$ \\
Health insurance $(n=353)$ & \\
Public (Medicare or Medicaid) & $47(43-53)$ \\
Private & $50(45-56)$ \\
No insurance & $2(1-4)$ \\
\hline & \\
\hline & \\
\hline &
\end{tabular}

Not all respondents provided complete information, number of respondents with nonmissing values noted for each item. Data shown reflect data for all respondents after multiple imputation of missing data. Percentages may not add to 100 due to rounding.

GED, General Educational Development certificate. 
Table 2. Pain Condition and Duration, Pain Levels, and Medication Use Among New York State Medical Cannabis Patients with Severe or Chronic Pain $(N=405)$

\begin{tabular}{|c|c|}
\hline Characteristic & $\%(95 \% \mathrm{Cl})$ \\
\hline \multicolumn{2}{|l|}{ Qualifying conditions $(n=405)^{\mathrm{a}}$} \\
\hline Neuropathy & $67(63-72)$ \\
\hline Inflammatory bowel disease & $19(15-22)$ \\
\hline Spinal cord injury & $18(14-22)$ \\
\hline Cancer & $15(12-19)$ \\
\hline HIV/AIDS & $3(1-5)$ \\
\hline Multiple sclerosis & $9(6-12)$ \\
\hline Amyotrophic lateral sclerosis & $0.2(0-0.7)$ \\
\hline Parkinson's disease & $1(0.3-3)$ \\
\hline \multicolumn{2}{|l|}{ How often do you experience pain? $(n=393)$} \\
\hline It's constant, always there & $80(76-84)$ \\
\hline At least once a day & $16(12-19)$ \\
\hline At least once a week & $3(2-5)$ \\
\hline Not every week, but at least once a month & $1(0.02-2)$ \\
\hline Duration of pain condition, years, mean $(n=390)$ & $10.8(9.8-11.7)$ \\
\hline \multicolumn{2}{|l|}{ Pain and interference (PEG) score $(n=388)$} \\
\hline Mild (0-3) & $9(6-12)$ \\
\hline Moderate (4-6) & $35(30-40)$ \\
\hline Severe $(7-10)$ & $56(51-61)$ \\
\hline \multicolumn{2}{|l|}{ Pain medication use in the past 30 days $(n=381)$} \\
\hline Opioid analgesics & $39(34-44)$ \\
\hline Acetaminophen & $17(13-21)$ \\
\hline Nonsteroidal anti-inflammatory drugs & $37(32-42)$ \\
\hline Tricyclic antidepressants & $4(2-6)$ \\
\hline Pregabalin & $8(5-11)$ \\
\hline Gabapentin & $17(13-21)$ \\
\hline Duloxetine & $9(6-12)$ \\
\hline Prescription patch or cream & $18(14-22)$ \\
\hline \multicolumn{2}{|l|}{ Number of medication types } \\
\hline 0 & $29(24-33)$ \\
\hline $1-2$ & $50(45-55)$ \\
\hline $3+$ & $21(17-25)$ \\
\hline $\begin{array}{l}\text { Days of opioid analgesic use in past month } \\
\text { among those reporting any use, mean }\end{array}$ & $24(22-26)$ \\
\hline
\end{tabular}

Not all respondents provided complete information, number of respondents with nonmissing values noted for each item. Data shown reflect data for all respondents after multiple imputation of missing data. Percentages may not add to 100 due to rounding.

${ }^{a}$ Respondents may report more than one condition

Neuropathy was the most common pain condition (67\%, 95\% CI 63-72\%) followed by inflammatory bowel disease (19\%, 95\% CI 15-22\%; Table 2). Most respondents reported constant pain (80\%, 95\% CI 76-84\%) and had scores indicating severe pain intensity and interference (56\%, 95\% CI 51-61\%). Of all respondents, 39\% (95\% CI 34-44\%) reported opioid analgesic use.

For medical cannabis, high THC (59\%, 95\% CI 54$64 \%)$ and balanced THC:CBD forms (54\%, 95\% CI $49-60 \%$, respectively) were used roughly equally, and sublingual tincture was the most common route of administration (57\%, 95\% CI 52-63\%; Table 3). Nearly half of respondents reported using more than one form (44\%, 95\% CI 39-50\%) and more than one route of administration (46\%, 95\% CI 41-51\%).
Nearly all respondents thought that the study described should be done (94\%, 95\% CI 92-97\%), and the vast majority reported that they would enroll if asked $(85 \%, 95 \%$ CI $81-88 \%$ definitely or probably yes; Table 4). Almost three-quarters would participate for 1 year or longer $(76 \%, 72-81 \%)$, and over half would respond to questions at least daily (59\%, 95\% CI 54-64\%). Increasing age was significantly associated with a lower willingness to participate $(-3$ percentage points per decade, $95 \% \mathrm{CI}-5$ to -0.3 ), lower willingness to participate for $\geq 1$ year $(-3$ percentage points per decade, $95 \% \mathrm{CI}-6$ to -0.2 ), and lower willingness to respond to questions at least daily ( -5 percentage points per decade, $95 \%$ CI -8 to -1 ; Table 4 ). Women were more likely than men to report being willing to respond to questions at least daily (12 percentage points, 95\% CI 2-22). There were no significant differences in willingness to participate by race/ ethnicity, education level, cancer status, pain and interference score, or use of opioid analgesics.

Among those willing to participate, 85\% (95\% CI $81-89 \%)$ wanted to participate because they felt that the research might help other people, and 79\% (95\% CI 74-83\%) felt that doctors need better scientific information about medical cannabis (Table 5). Of respondents not willing to participate, $28 \%$ (95\% CI 14 $42 \%$ ) reported that the study would take too much of their time, and $28 \%$ (95\% CI $13-42 \%)$ reported that they do not want to provide medical information to researchers.

\section{Discussion}

While patient groups, clinicians, and policymakers have called for more research on medical cannabis, to our knowledge, this is the first study that has examined willingness of people with chronic pain to participate in such research. Not only did virtually all respondents support the proposed study but also a vast majority reported that they would enroll if asked. However, with an estimated survey response rate of $30 \%$, willingness to participate in the study described may be as low as $26 \%(85 \% \times 30 \%)$ of the broader population. Our findings suggest that long-term studies involving repeated, frequent data collection are of interest to the target population and recruitment would be feasible.

Our findings also suggest that future studies can successfully recruit patients who take opioid analgesics, a subgroup of particular clinical importance. Such patients can have ambivalence about long-term use of opioid analgesics, ${ }^{47-49}$ want research studies to 
Table 3. Medical Cannabis Use Among New York State Medical Cannabis Patients with Severe or Chronic Pain $(N=405)$

\begin{tabular}{lc} 
Characteristic & $\%(95 \% \mathrm{Cl})$ \\
\hline $\begin{array}{l}\text { Duration of medical cannabis use } \\
\text { in months, mean }(n=352)\end{array}$ & $5.1(4.7-5.4)$ \\
$\begin{array}{l}\text { Number of days of medical cannabis use } \\
\text { in past month, mean }(n=359)\end{array}$ & $21(19-22)$ \\
$\begin{array}{l}\text { Dosage form }(n=370) \\
\quad \text { High THC }\end{array}$ & \\
$\quad$ Balanced THC:CBD & $59(54-64)$ \\
$\quad$ High CBD & $54(49-60)$ \\
$\quad$ More than one dosage form & $37(32-42)$ \\
Route of administration $(n=370)$ & $44(39-50)$ \\
Sublingual tincture & \\
Oral capsule or oil & $57(52-63)$ \\
Vapor oil & $29(24-34)$ \\
More than one route of administration & $64(59-69)$ \\
\hline
\end{tabular}

Not all respondents provided complete information, number of respondents with nonmissing values is noted for each item. Data shown reflect data for all respondents after multiple imputation of missing data. Percentages may not add to 100 due to rounding.

CBD, cannabidiol; THC, tetrahydrocannabinol. include decreasing opioid dose as an end-point, ${ }^{50}$ and may seek medical cannabis as an alternative or adjunctive therapy. ${ }^{51}$ As suggested by surveys and anecdotal evidence, patients may also seek medical cannabis for the purposes of tapering or discontinuing opioid analgesics. ${ }^{52-59}$ State-level ecological studies have found associations between medical cannabis laws and lower rates of opioid prescribing and overdoses involving opioids. ${ }^{60-62}$ In the era of the opioid epidemic, rigorous patient-level studies of the impact of medical cannabis use on opioid analgesic use are urgently needed.

While patient populations and study designs are distinct, our finding of lower willingness to participate with increasing age is consistent with some, but not all, recent surveys about observational (e.g., biobanking and genomics) and interventional (e.g., clinical trial) research. ${ }^{19,20,23,28,30,45,63}$ The difference by age that we found may be due, at least in part, to the mode of data collection (phone-based), but previous surveys about technology-based studies have found mixed associations between age and willingness to participate. ${ }^{31,32,46}$ Older

Table 4. Willingness to Participate in a Longitudinal Cohort Study Among New York State Medical Cannabis Patients with Severe or Chronic Pain

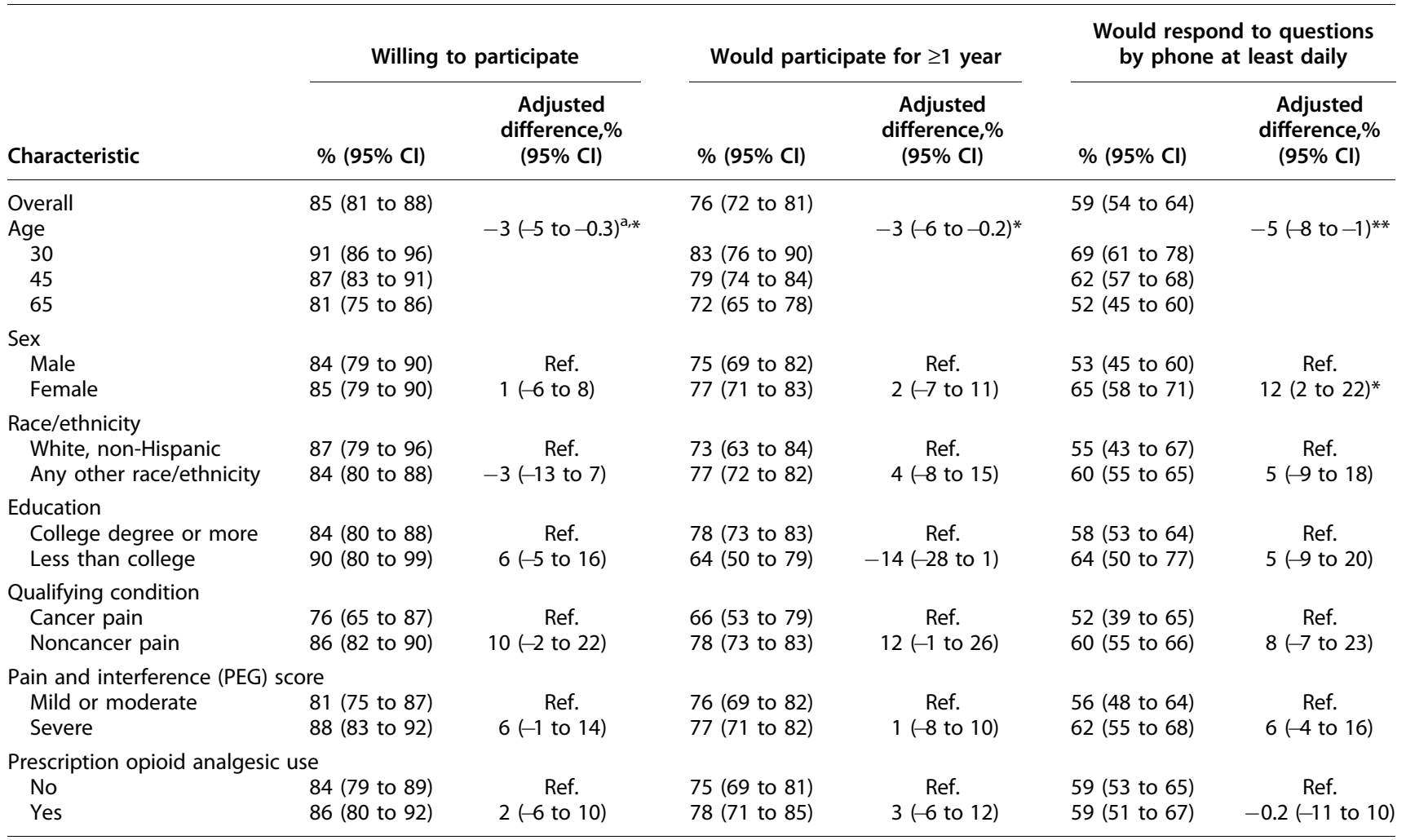

Values are regression-adjusted for all characteristics listed in this table.

${ }^{a}$ Refers to the difference with a10-year change in age.

${ }^{*} p<0.05 ;{ }^{* *} p<0.01$. 
Table 5. Reasons for Being Willing, or Not Willing, to Participate in Research Among New York State Medical Cannabis Patients with Severe or Chronic Pain $(N=405)$

$\%(95 \% \mathrm{Cl})$

\begin{tabular}{ll}
\hline Reasons for being willing to participate among those willing \\
Research might help other people & $85(81-89)$ \\
Doctors need better scientific information & $79(74-83)$ \\
about medical cannabis & $76(71-81)$ \\
Research might help me & $62(56-67)$ \\
I want to contribute to scientific research & $28(23-33)$ \\
I might get money for participating & $16(12-20)$ \\
My doctor would want me to participate & $15(11-19)$ \\
My family or friends would want me to participate & $28(14-42)$ \\
Reasons for not being willing to participate among those not willing \\
The study would take too much of my time & $28(13-42)$ \\
I don't want to provide my medical information & \\
to researchers & $27(13-41)$ \\
I don't want to provide samples (urine or blood) & \\
$\quad$ for lab tests & $21(8-34)$ \\
I don't want researchers to ask me personal questions & $20(7-32)$ \\
I want more information about the study & $18(6-31)$ \\
I don't trust researchers to protect my privacy & $16(5-28)$ \\
There is not enough money compensation for my time & $15(4-26)$ \\
I want more information about who the researchers are & $15(1-21)$ \\
The study won't help me directly & $10(0.4-20)$ \\
I don't want researchers to know I take & $0(0)$ \\
medical cannabis & \\
The study won't help anyone &
\end{tabular}

respondents may also have differing attitudes about research (e.g., concern for experimentation) or face other potential barriers. ${ }^{23}$ To ensure equal representation, future studies of chronic pain and medical cannabis should specifically seek to engage older adults.

While we designed our study description based on previously described information needs of potential study participants with chronic pain, ${ }^{17,18}$ there are several factors which could improve willingness to participate even further. About one third of respondents who were not willing to participate reported wanting to know more information on the study. This may include more information about access to clinicians during the study, more details about the time burden of the study, more information about management of increased pain or adverse events, or even details such as the names and credentials of the investigators. ${ }^{17}$ Willingness to participate may also increase with the offer of personalized feedback at the end of the study (e.g., a report on pain levels and cannabis use), a feature that other potential research participants have reported valuing. ${ }^{19}$

This study has several limitations. First, as we recruited a convenience sample, respondents' views may not be representative of the broader population with chronic pain who take medical cannabis. We could not assess demographic differences between sur- vey respondents and the broader New York medical cannabis patient population because New York has not released these data. Furthermore, as New York's medical cannabis program only distributes extracts that are much more expensive than plant material, eligible patients in New York may have different sociodemographic characteristics than potentially eligible patients in other states. Second, we did not have exact numbers of eligible patients, and our estimated number (and response rate) may not be accurate. Third, we asked about willingness to participate in a hypothetical study, responses to invitations to enroll in an actual study may be different. Finally, we relied on self-report for cannabis and opioid analgesic use and did not confirm with medical records.

\section{Conclusion}

In conclusion, we found high levels of support for a longitudinal cohort study with frequent data collection among patients with chronic pain who take medical cannabis. To the extent that future studies can reach a broad selection of patients, our findings also suggest that recruitment of representative samples is possible. While access to medical cannabis has expanded greatly, interventional research on the efficacy of cannabis for pain is still limited due to funding and regulations. Strong observational studies have the potential to fill in evidence gaps, and the current study demonstrates that patients are willing to participate.

\section{Acknowledgments}

This study was funded, in part, by a grant from the National Institute on Drug Abuse of the National Institutes of Health (K24DA036955). The funding agency had no role in design of the study, collection, analysis, and interpretation of data, or in writing the article.

\section{Author Disclosure Statement}

No competing financial interests exist.

\section{References}

1. Guy GP, Jr., Zhang K, Bohm MK, et al. Vital signs: changes in opioid prescribing in the United States, 2006-2015. MMWR Morb Mortal Wkly Rep. 2017;66:697-704.

2. Rudd RA, Seth $P$, David F, et al. Increases in drug and opioid-involved overdose deaths-United States, 2010-2015. MMWR Morb Mortal Wkly Rep. 2016;65:1445-1452.

3. National Academies of Sciences Engineering and Medicine. The health effects of cannabis and cannabinoids: current state of evidence and recommendations for research. Washington, DC: The National Academies Press, 2017.

4. DEA Announces Actions Related To Marijuana and Industrial Hemp. 2016. Available at https://www.dea.gov/divisions/hq/2016/hq081116.shtml (last Accessed November 21, 2017). 
5. Abrams DI, Jay CA, Shade SB, et al. Cannabis in painful HIV-associated sensory neuropathy: a randomized placebo-controlled trial. Neurology. 2007;68:515-521.

6. Ellis RJ, Toperoff W, Vaida F, et al. Smoked medicinal cannabis for neuropathic pain in HIV: a randomized, crossover clinical trial. Neuropsychopharmacology. 2009;34:672-680.

7. Wallace MS, Marcotte TD, Umlauf A, et al. Efficacy of inhaled cannabis on painful diabetic neuropathy. J Pain. 2015;16:616-627.

8. Wilsey B, Marcotte T, Deutsch R, et al. Low-dose vaporized cannabis sig nificantly improves neuropathic pain. J Pain. 2013;14:136-148.

9. Wilsey B, Marcotte T, Tsodikov A, et al. A randomized, placebo-controlled, crossover trial of cannabis cigarettes in neuropathic pain. J Pain. 2008;9: 506-521.

10. Noyes R, Jr., Brunk SF, Avery DA, et al. The analgesic properties of delta-9tetrahydrocannabinol and codeine. Clin Pharmacol Ther. 1975;18:84-89.

11. Cruise CE, Broderick J, Porter $L$, et al. Reactive effects of diary selfassessment in chronic pain patients. Pain. 1996;67:253-258.

12. von Baeyer CL. Reactive effects of measurement of pain. Clin J Pain. 1994 10:18-21.

13. Robins JM, Hernan MA, Brumback B. Marginal structural models and causal inference in epidemiology. Epidemiology. 2000;11:550-560.

14. Ryan J, Sharts-Hopko N. The experiences of medical marijuana patients: a scoping review of the qualitative literature. J Neurosci Nurs. 2017;49:185-190.

15. Satterlund TD, Lee JP, Moore RS. Stigma among California's medical marijuana patients. J Psychoactive Drugs. 2015;47:10-17.

16. New York State Department of Health. Medical use of marijuana under the Compassionate Care Act: two-year report. Albany, NY: New York State Department of Health, 2016.

17. Casarett D, Karlawish J, Sankar P, et al. Obtaining informed consent for clinical pain research: patients' concerns and information needs. Pain. 2001;92:71-79.

18. Casarett D, Karlawish J, Sankar P, et al. Obtaining informed consent for cancer pain research: do patients with advanced cancer and patients with chronic pain have different concerns? J Pain Symptom Manage. 2002;24: 506-516.

19. Kaufman DJ, Baker R, Milner LC, et al. A survey of U.S adults' opinions about conduct of a nationwide precision medicine initiative $(R)$ cohort study of genes and environment. PLoS One. 2016;11:e0160461.

20. Sanderson SC, Brothers KB, Mercaldo ND, et al. Public attitudes toward consent and data sharing in Biobank Research: a large multi-site experimental survey in the US. Am J Hum Genet. 2017;100:414-427.

21. Rahm AK, Wrenn M, Carroll NM, et al. Biobanking for research: a survey of patient population attitudes and understanding. J Community Genet. 2013;4:445-450.

22. Horch JD, Carr EC, Harasym P, et al. Firefighter willingness to participate in a stem cell clinical trial for burns: a mixed methods study. Burns. 2016; 42:1740-1750

23. Mao JJ, Tan T, Li SQ, et al. Attitudes and barriers towards participation in an acupuncture trial among breast cancer patients: a survey study. BMC Complement Altern Med. 2014;14:7.

24. Kaplan CP, Napoles AM, Narine $S$, et al. Knowledge and attitudes regarding clinical trials and willingness to participate among prostate cancer patients. Contemp Clin Trials. 2015;45:443-448.

25. Geppert $C$, Candilis $P, B a k e r ~ S$, et al. Motivations of patients with diabetes to participate in research. AJOB Empir Bioeth. 2014;5:14-21.

26. Slegers $C$, Zion D, Glass D, et al. Why do people participate in epidemiological research? J Bioeth Inq. 2015;12:227-237.

27. Locock L, Smith L. Personal benefit, or benefiting others? Deciding whether to take part in clinical trials. Clin Trials. 2011;8:85-93.

28. Walker ER, Nelson CR, Antoine-LaVigne D, et al. Research participants' opinions on genetic research and reasons for participation: a Jackson Heart Study focus group analysis. Ethn Dis. 2014;24:290-297.

29. Robinson JO, Carroll TM, Feuerman LZ, et al. Participants and study decliners' perspectives about the risks of participating in a clinical trial of whole genome sequencing. J Empir Res Hum Res Ethics. 2016;11:21-30

30. Dube K, Evans D, Sylla L, et al. Willingness to participate and take risks in HIV cure research: survey results from 400 people living with HIV in the US. J Virus Erad. 2017;3:40-50.e21.

31. James DC, Harville $C$, 2nd. Barriers and motivators to participating in mHealth research among African American men. Am J Mens Health. 2017; 11:1605-1613.
32. James DC, Harville C, 2nd, Whitehead N, et al. Willingness of African American women to participate in e-Health/m-Health research. Telemed J E Health. 2016;22:191-197.

33. Moorcraft SY, Marriott C, Peckitt C, et al. Patients' willingness to participate in clinical trials and their views on aspects of cancer research: results of a prospective patient survey. Trials. 2016;17:17.

34. Spears CR, Sandberg JC, O'Neill JL, et al. Recruiting underserved mothers to medical research: findings from North Carolina. J Health Care Poor Underserved. 2013;24:1801-1815.

35. Byrne MM, Tannenbaum SL, Gluck S, et al. Participation in cancer clinical trials: why are patients not participating? Med Decis Making. 2014;34: 116-126.

36. Kratz AL, Kalpakjian CZ, Hanks RA. Are intensive data collection methods in pain research feasible in those with physical disability? A study in persons with chronic pain and spinal cord injury. Qual Life Res. 2017;26: 587-600.

37. Sterling R, Henderson GE, Corbie-Smith G. Public willingness to participate in and public opinions about genetic variation research: a review of the literature. Am J Public Health. 2006;96:1971-1978.

38. Ellis PM. Attitudes towards and participation in randomised clinical trials in oncology: a review of the literature. Ann Oncol. 2000;11:939-945.

39. Krebs EE, Lorenz KA, Bair MJ, et al. Development and initial validation of the PEG, a three-item scale assessing pain intensity and interference. J Gen Intern Med. 2009;24:733-738.

40. Kroenke K, Theobald D, Wu J, et al. Comparative responsiveness of pain measures in cancer patients. J Pain. 2012;13:764-772.

41. White IR, Royston P, Wood AM. Multiple imputation using chained equations: issues and guidance for practice. Stat Med. 2011;30:377399.

42. Lanfear DE, Jones PG, Cresci S, et al. Factors influencing patient willingness to participate in genetic research after a myocardial infarction. Genome Med. 2011;3:39.

43. Cottler LB, McCloskey DJ, Aguilar-Gaxiola S, et al. Community needs, concerns, and perceptions about health research: findings from the clinical and translational science award sentinel network. Am J Public Health. 2013;103:1685-1692.

44. Uhlmann S, Milloy MJ, Ahamad K, et al. Factors associated with willingness to participate in a pharmacologic addiction treatment clinical trial among people who use drugs. Am J Addict. 2015;24:368-373.

45. Kerath SM, Klein G, Kern M, et al. Beliefs and attitudes towards participating in genetic research-a population based cross-sectional study. BMC Public Health. 2013;13:114.

46. Green BB, Anderson ML, Ralston JD, et al. Patient ability and willingness to participate in a web-based intervention to improve hypertension control. J Med Internet Res. 2011;13:e1.

47. Brooks EA, Unruh A, Lynch ME. Exploring the lived experience of adults using prescription opioids to manage chronic noncancer pain. Pain Res Manag. 2015;20:15-22.

48. Simmonds MJ, Finley EP, Vale S, et al. A qualitative study of veterans on long-term opioid analgesics: barriers and facilitators to multimodality pain management. Pain Med. 2015;16:726-732.

49. Howe CQ, Sullivan MD, Saunders KW, et al. Depression and ambivalence toward chronic opioid therapy for chronic noncancer pain. Clin J Pain. 2012;28:561-566.

50. Casarett D, Karlawish J, Sankar P, et al. Designing pain research from the patient's perspective: what trial end points are important to patients with chronic pain? Pain Med. 2001;2:309-316.

51. Zaller N, Topletz A, Frater S, et al. Profiles of medicinal cannabis patients attending compassion centers in Rhode island. J Psychoactive Drugs. 2015;47:18-23.

52. Lucas P. Cannabis as an adjunct to or substitute for opiates in the treatment of chronic pain. J Psychoactive Drugs. 2012;44:125-133.

53. Piper BJ, DeKeuster RM, Beals ML, et al. Substitution of medical cannabis for pharmaceutical agents for pain, anxiety, and sleep. J Psychopharmacol. 2017:31:569-575.

54. Boehnke KF, Litinas E, Clauw DJ. Medical cannabis use is associated with decreased opiate medication use in a retrospective cross-sectional survey of patients with chronic pain. J Pain. 2016;17:739-744.

55. Reiman A, Welty M, Solomon P. Cannabis as a substitute for opioid-based pain medication: patient self-report. Cannabis Cannabinoid Res. 2017;2: 160-166. 
56. Lucas P, Walsh Z. Medical cannabis access, use, and substitution for prescription opioids and other substances: a survey of authorized medical cannabis patients. Int J Drug Policy. 2017;42:30-35.

57. Vigil JM, Stith SS, Adams IM, et al. Associations between medical cannabis and prescription opioid use in chronic pain patients: a preliminary cohort study. PLoS One. 2017;12:e0187795.

58. Corroon JM, Jr., Mischley LK, Sexton M. Cannabis as a substitute for prescription drugs-a cross-sectional study. J Pain Res. 2017;10:989-998.

59. Sexton M, Cuttler C, Finnell JS, et al. A cross-sectional survey of medical cannabis users: patterns of use and perceived efficacy. Cannabis Cannabinoid Res. 2016;1:131-138.

60. Bachhuber MA, Saloner B, Cunningham CO, et al. Medical cannabis laws and opioid analgesic overdose mortality in the United States, 19992010. JAMA Intern Med. 2014;174:1668-1673.

61. Bradford AC, Bradford WD. Medical marijuana laws reduce prescription medication use in Medicare part D. Health Aff (Millwood). 2016;35: 1230-1236.

62. Bradford AC, Bradford WD. Medical marijuana laws may be associated with a decline in the number of prescriptions for Medicaid enrollees. Health Aff (Millwood). 2017;36:945-951.
63. Stuchlik P, Zhang X, Gavin S, et al. Willingness to participate in cardiovascular clinical research among African-Americans. J Clin Res Bioeth. 2015;6:pii:223

Cite this article as: Bachhuber MA, Arnsten JH, Starrels JL, Cunningham CO (2018) Willingness to participate in longitudinal research among people with chronic pain who take medical cannabis: a crosssectional survey, Cannabis and Cannabinoid Research 3:1, 45-53, DOI: 10.1089/can.2017.0051.

\section{Abbreviations Used}

$\mathrm{CBD}=$ cannabidiol

$\mathrm{RCT} s=$ randomized controlled trials $\mathrm{THC}=$ tetrahydrocannabinol

\section{Publish in Cannabis and Cannabinoid Research}

Cannabis and

Cannabinoid

Research
- Immediate, unrestricted online access

- Rigorous peer review

- Compliance with open access mandates

- Authors retain copyright

- Highly indexed

- Targeted email marketing 